\title{
Percepciones de una muestra de adolescentes ecuatorianos sobre el apoyo social, la comunicación y la cohesión en sus familias. Diferencias por edad y sexo e implicaciones para la educación emocional ${ }^{1}$
}

\author{
Beatriz Álvarez-González \\ Universidad Nacional de Educación a Distancia-UNED, España \\ mail: balvarezdedu.uned.es \\ ORCID: https://orcid.org/0000-0002-2003-1117
}

\author{
Luz Ivonne Zabaleta \\ Universidad Técnica Particular de Loja, Ecuador / Programa de \\ Doctorado en Educación (EIDUNED) \\ mail: lizabaleta@utpl.edu.ec \\ ORCID: https://orcid.org/0000-0002-8109-4760
}

\section{RESUMEN}

La adolescencia es un proceso de transición con cambios físicos, cognoscitivos y psicosociales (Extremera y Fernández-Berrocal, 2004; Rodríguez-Fernández et al. 2016). El desarrollo en esta etapa recibe la influencia de los pares, y la de la propia familia. Para profundizar en estas interacciones y en las dinámicas familiares resulta útil contar con instrumentos que evalúen las relaciones interpersonales, dando una estimación, no solo de los niveles de competencias emocionales, sino del perfil social. Por ello, esta investigación se centra en recabar las percepciones de una muestra de adolescentes ecuatorianos (Loja) mediante las escalas del Cuestionario Cómo es Tu Familia (CTF, Hernández et al. 1996), que plantean situaciones del contexto familiar para las que se considera que la persona debe contar con determinadas competencias emocionales, en las respuestas. Las respuestas se analizaron en función de dos variables moduladoras, edad y sexo. Del CTF se seleccionaron las escalas correspondientes a tres factores de los siete del cuestionario, concretamente: factor A Tipo de relaciones: Cohesión familiar, Participación en la solución de problemas, y Comunicación hijos/as-progenitores. Los resultados se han analizado mediante un estudio correlacional e inferencial, estableciendo comparaciones entre sexos, y para dos rangos de edad: 12-14 y 15-17 años. Con respecto a las edades que abarca el cuestionario CTF, este podría contribuir en los estudios de pre y postest mediante los que se evalúa los desarrollos logrados a través de programas de educación emocional.

Palabras Clave: apoyo social, comunicación familiar, cohesión familiar, adolescentes, educación emocional, diferencias por edad y sexo.

\section{A sample of Ecuadorian adolescents' perceptions on social support and family communication and cohesion. Differences between age and sex, and implications for emotional education}

\section{ABSTRACT}

Adolescence is a transition process with physical, cognitive and psychosocial changes (Extremera y Fernández-Berrocal, 2004; RodríguezFernández et al. 2016). Development at this stage is influenced by peers, and by the family itself. In order to deepen in these interactions and family dynamics, it is useful to count on instruments to evaluate interpersonal relationships, giving an estimate, not only on the levels of emotional competencies, but also on the social profile. For this reason, this research focuses on collecting the perceptions of a sample of Ecuadorian adolescents (Loja) using the scales of the Questionnaire Cómo es Tu Familia (CTF, Hernández et al. 1996), which pose situations in the family context for which it is considered that the person must have certain emotional competencies. The responses were analyzed according to two modulating variables: age and sex. From the CTF, the scales corresponding to three of the seven factors in the questionnaire were selected, namely factor A Type of relationships: Family cohesion, Participation in problem solving, and Childparent communication. The results were analyzed through a correlational and inferential study, establishing comparisons between sexes, and for two age ranges: 12-14 and 15-17 years old. With regard to the ages covered by CTF, this could contribute to pre- and posttest studies, where achievements through emotional education programs are evaluated.

Keywords: Social support, family communication, family cohesion, adolescents, emotional education, age and sex differences.

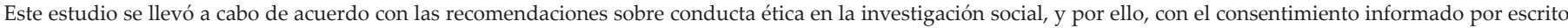
de todas las familias y la autorización de los centros educativos que participaron en la presente investigación.

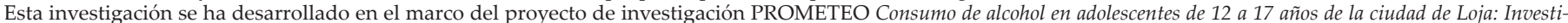

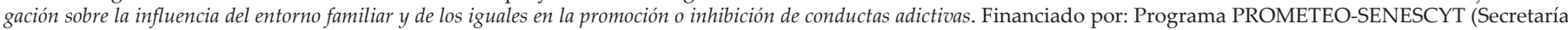
Nacional de Ciencia y Tecnología)

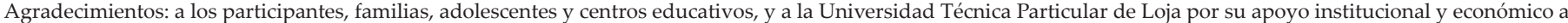
esta investigación

ISSN: 0210-2773

DOI: https://doi.org/10.17811/rifie.50.4.2021.787-798 


\section{Introducción}

La adolescencia es un proceso de transición hacia la edad adulta, y este desarrollo, según diversos estudios (Extremera y Fernández-Berrocal, 2004) recibe la influencia de los pares, y la de la propia familia. Con respecto a la familia, se ha constatado que, si se presentan deficiencias en la crianza familiar, los adolescentes pueden adquirir estrategias desadaptativas a la hora de enfrentarse a los problemas, y esto influirá negativamente en su manejo de las situaciones estresantes (Rodrigo et al. 2008). Puede implicar situaciones de crisis, por los cambios que experimenta la persona, pero también va acompañada del desarrollo de habilidades que serán fundamentales para que la persona se inserte en la vida social. Hay que tener en cuenta que, a lo largo de la adolescencia, la familia puede atravesar situaciones problemáticas y estresantes, que pueden tener efectos de distinto signo en diversos aspectos de la persona, como en la capacidad para afrontar los problemas (Aunola, Stattin y Nurmi; 2000 Rodrigo et al., 2008) o el apoyo social (Méndez y Barra, 2008). Por otro lado, la comunicación familiar es fundamental para sus miembros ya que permite mantener las condiciones de los sistemas familiares. La influencia del contexto familiar en el desarrollo de las competencias emocionales de hijos e hijas se constata en diversos estudios, y como esta influencia es clave para el ajuste psicosocial de la persona (Gracia, Lila y García, 2008).

Los estudios que se vienen desarrollando sobre inteligencia emocional (IE) y sobre competencias emocionales, desde hace más de dos décadas, vienen a coincidir en la importancia, por un lado, de continuar profundizando en el constructo de IE para avanzar en el desarrollo de herramientas y estrategias eficaces en la medición de la IE (Extremera y Fernández-Berrocal, 2004, 2005, Fuentes-Aguilar y Merino-Escobar, 2016), por otro, seguir implementando programas de educación emocional, tanto para trabajar la prevención, dando respuesta a las necesidades del alumnado de todos los niveles educativos, como para atender mediante estos programas a la diversidad de dicho alumnado (Cepa, Heras y Fernández-Hawrylak, 2017).

En estos programas de intervención, los instrumentos más frecuentes para la evaluación son los autoinformes, y en lo que respecta a los contenidos de estos, los más utilizados miden rasgos de personalidad (Pena y Repetto, 2008; Joseph, Jin, Newman y O'Boyle, 2015), mientras que otros se centran en habilidades como el autocontrol, la consciencia social y el desarrollo de actividades sociales (Clark, 2006). No obstante, e independientemente del modelo seguido, muchos de estos instrumentos se dirigen a una única población (infancia, adolescencia, padres, madres, profesorado).

Sin embargo, para profundizar en las dinámicas familiares resulta útil contar con instrumentos que evalúen las relaciones interpersonales, mediante versiones para diversos informantes (progenitores, hijos e hijas, profesorado, en su caso) de esta forma, se reduce en parte el sesgo de la autopercepción, al introducir la versión de más de un informante sobre la misma dinámica, lo que permite también comprobar el grado de acuerdo de las distintas percepciones respecto a una misma situación familiar. Además, se proporciona una estimación, no solo del nivel de IE, sino del perfil social (Fernández-Berrocal et al., 2012). Este es el caso de la escala utilizada en el estudio de Polo del Río et al. (2012), Escala de Afecto versión hijos, EA-H (Bersabé, Fuentes y Motrico, 2001), así como de la utilizada en esta investigación: Cómo es TU familia (CTF) versión adolescentes (Hernández et al., 1996).

Otros estudios se centran en la influencia de elementos de la parentalidad que promueven el desarrollo social de los adolescentes, y entre estos se destacan los que interesan en este estudio: afrontamiento, toma de decisiones y resolución de problemas (Martínez-González, 2009, Ciocanel et al., 2017). Los resultados de estos últimos estudios plantean la necesidad de la formación en estrategias adaptativas, dirigidas tanto a padres y educadores como a las personas más jóvenes (Mestre, 2014).

Además de contar con instrumentos eficaces para evaluar el perfil y nivel de desarrollo de las competencias emocionales, también es necesario el desarrollo de programas de intervención en educación emocional, y para ello hay que entender qué se entiende por inteligencia emocional (IE) y por competencias emocionales. A este respecto, (Bar-On, 2000; Grewal y Salovey, 2006, Bisquerra, y Pérez-Escoda, 2007) conceptualizan la IE como el conjunto de las habilidades personales, emocionales y sociales que determinan la capacidad de las personas para afrontar las presiones del entorno. En este sentido, Valiente et al. (2020) han constatado que los adolescentes controlan mejor las situaciones de estrés cuando cuentan con competencias que les permiten potenciar sus emociones positivas y reducir las negativas, adaptándose mejor así a los cambios y las adversidades.

Garaigordobil (2018) en su estudio sobre inteligencia emocional intrapersonal durante la adolescencia, explora las variables de sexo y edad para identificar posibles diferencias y su vinculación con otras variables y predictores, dado que estas posibles asociaciones se consideran relevantes para el diseño de programas de desarrollo de competencias emocionales. A través de estos programas es posible desarrollar y reforzar las competencias emocionales que permiten a los y las adolescentes afrontar de manera adaptativa las situaciones problemáticas que se puedan presentar en sus familias. Estas competencias emocionales tienen un elevado valor para la vida y pueden ser aprendidas (Bar-On y Parker, 2000; Salovey y Sluyter, 1997, Sanmartín, Gonzálevez, y Vicent, 2018, Pérez Escoda y Filella Guiu, 2019)

En esta investigación se busca comprobar la influencia de dos variables moduladoras, edad y sexo, en las respuestas de una muestra de adolescentes ecuatorianos (Loja) a las escalas del Cuestionario CTF, que plantean situaciones del contexto familiar para las que se considera que la persona, en este caso el/la adolescente debe contar con determinadas competencias emocionales. Para ello, se seleccionaron las escalas correspondientes a tres factores de los siete que componen el CTF, concretamente: del factor A Tipo de relaciones: Cohesión familiar, Participación en la solución de problemas, y Comunicación hijos/as-progenitores, (tabla 3). Y se han analizado mediante un estudio correlacional e inferencial, estableciendo comparaciones entre sexos, y para dos rangos de edad: 12-14 y 15-17 años.

La razón de realizar contrastes por sexo se basa en diversos estudios previos que han comprobado la importancia de las competencias emocionales como factor de protección de conductas de riesgo en la adolescencia, en este sentido Fernández Berrocal et al. (2012) se plantearon si las mujeres superaban a los hombres en competencia emocional, y encontraron que las diferencias halladas en este sentido estaban mediadas por la edad, otra variable que se considera fundamental en el desarrollo de las competencias emocionales.

En cuanto al interés por utilizar las escalas del Cuestionario $\mathrm{CTF}$, se debe a que están centradas en las dinámicas de comunicación e interacción del contexto familiar, también se parte de estudios previos como el de Sánchez-Nuñez y Latorre-Postigo (2012) que abordaron la inteligencia emocional autoinformada (IEA) y el clima familiar, utilizando para este último aspecto la "Escala de Clima Familiar" de Moos, Moos y Trickett (1995), midiendo también las percepciones de los hijos sobre la inteligencia 
emocional de los padres (IEP). Entre los principales resultados vieron que la IEA y la IEP predecían la expresividad en el entorno familiar. Por otro lado, responden a planteamientos desarrollados en el contexto de América Latina (Ministerio de Educación del Ecuador, 2020; Álvarez-González y Zabaleta, 2020)

\section{Objetivos}

A partir de la revisión de la bibliografía desarrollada en torno al tema de estudio, se plantearon dos objetivos generales, por un lado: analizar las percepciones de una muestra de adolescentes con respecto a sus relaciones familiares para inferir a través de estas si los y las adolescentes de la muestra tienen un perfil diferenciado con respecto a las variables (5 escalas de CTF) de: cohesión familiar, participación en la solución de problemas, afrontamiento de problemas y comunicación con el padre y la madre, en función del sexo y del grupo de edad. Y por otro: comprobar si aparecen diferencias significativas por sexo y grupo de edad en variables relevantes para la educación emocional.

Estos objetivos se abordaron a través de otros más específicos, y son: identificar si aparecen diferencias significativas por rangos de edad: 12-14 y 15-17 años en las respuestas de la muestra de adolescentes; identificar si aparecen diferencias significativas por sexo en las respuestas de la muestra de adolescentes, y por último, identificar si aparecen diferencias significativas en las cinco escalas del CTF comparando las respuestas en función de sexo y edad.

\section{Método}

\section{Diseño de estudio}

Participantes

En este estudio participó un total de 921 adolescentes entre 12 y 17 años sin problemáticas específicas. La edad media es de 14.7 años, con desviación estándar de \pm 1.61 años. Exceptuando los casos con 12 años, el resto de edades están representadas en porcentajes muy similares (tabla_1). Por sexos, la muestra está equilibrada: $52.3 \%$ hombres y $47.7 \%$ mujeres. Los centros se seleccionaron mediante muestreo no probabilístico, por conveniencia o incidental, y las aulas fueron seleccionadas por los equipos directivos de los centros, y eran completas en cada grado, $8^{\circ} \mathrm{E}-$ GB-3ํㅡㄹ Bachillerato del Sistema Educativo Ecuatoriano (tabla_2). Para la selección de la muestra se siguió el protocolo de contactar a las familias y adolescentes mediante carta de presentación y consentimiento informado.

Se contactó con los colegios a través de la Escuela de Psicología de la Universidad, primero con los equipos directivos. Se les informó sobre las edades y niveles educativos objeto de la

Tabla 1.

Población y muestra del estudio

\begin{tabular}{lc}
\hline \multicolumn{1}{c}{ Población y muestra } \\
\hline $\begin{array}{l}\text { Población del estudio (12-17 años) } \\
\text { (adolescentes y preadolescentes) }\end{array}$ & 17000 \\
Muestra invitada & 2000 \\
Muestra aceptante & 1200 \\
Muestra productora de datos & 921 \\
\hline
\end{tabular}

investigación, y estos equipos establecieron la planificación para los aplicadores. El equipo investigador visitó un colegio por día. Dos encuestadores visitaban cada aula y la aplicación de los instrumentos era simultánea en las seis aulas que el colegio asignaba a este estudio. La aplicación duraba aproximadamente entre 45 y 60 minutos, ya que, por necesidad de la investigación, respondían a tres cuestionarios simultáneamente, un cuestionario sociodemográfico, el CTF y el Test Alcohol Use Disorders Identification (AUDIT).

\section{Población y muestra del estudio}

La muestra aceptante se distribuyó en cinco colegios de carácter público, concertado y privado, y el número de estudiantes por niveles y centros se muestran en la tabla 2.

Tabla 2.

Características de la muestra

\begin{tabular}{|c|c|c|c|}
\hline \multicolumn{4}{|c|}{ Características de la muestra. - N=921 - } \\
\hline \multicolumn{2}{|c|}{ VARIABLE / Categoría } & \multicolumn{2}{|c|}{ Descriptivos } \\
\hline \multirow[t]{8}{*}{$E D A D$ (años) } & Media (Desv. Est.) & $14.7( \pm$ & .61) \\
\hline & Rango (mediana) & & / 17 (15.0) \\
\hline & 12 años & 102 & $(11.1 \%)$ \\
\hline & 13 años & 150 & $(16.3 \%)$ \\
\hline & 14 años & 178 & $(19.3 \%)$ \\
\hline & 15 años & 176 & $(19.1 \%)$ \\
\hline & 16 años & 155 & $(16.8 \%)$ \\
\hline & 17 años & 160 & $(17.7 \%)$ \\
\hline \multirow[t]{2}{*}{ SEXO } & Hombre & 482 & $(52.3 \%)$ \\
\hline & Mujer & 439 & $(47.7 \%)$ \\
\hline \multirow[t]{5}{*}{ COLEGIOC1 } & & 224 & $(24.3 \%)$ \\
\hline & C2 & 219 & $(23.8 \%)$ \\
\hline & C3 & 174 & $(18.9 \%)$ \\
\hline & $C 4$ & 168 & $(18.2 \%)$ \\
\hline & C5 & 136 & $(14.8 \%)$ \\
\hline \multirow[t]{3}{*}{ TIPO DE COLEGIO } & Privado & 528 & $(57.3 \%)$ \\
\hline & Concertado & 219 & $(23.8 \%)$ \\
\hline & Público & 174 & $(18.9 \%)$ \\
\hline \multirow[t]{6}{*}{ CURSO } & 8o Básica & 146 & $(15.6 \%)$ \\
\hline & 9ํo Básica & 168 & $(18.2 \%)$ \\
\hline & 10º Básica & 182 & $(19.8 \%)$ \\
\hline & $1^{\underline{o}}$ Bachillerato & 175 & $(19.0 \%)$ \\
\hline & $2^{\circ}$ Bachillerato & 155 & $(16.8 \%)$ \\
\hline & $3^{o}$ Bachillerato & 95 & $(10.3 \%)$ \\
\hline
\end{tabular}




\section{Procedimiento}

Para esta investigación se ha utilizado el cuestionario CTF, (Hernández et al., 1996), que constituye una de las dos versiones incluidas en el Manual de Familia y Adolescencia: Indicadores de Salud. Versión para adolescentes (CTF), y versión padres, Cómo es SU familia (CSF). La estructura del CTF se presenta en la tabla_2, y se seleccionó porque se trata de un cuestionario que recaba datos, a través de las percepciones de adolescentes y familias sobre las dinámicas de afrontamiento de problemas y comunicación intrafamiliar, en sus propios entornos familiares. Hay estudios que señalan la necesidad de contar con instrumentos centrados en el estudio del funcionamiento familiar (Zicavo, Palma y Ga- rrido, 2012). Estos autores asumen el funcionamiento familiar como las pautas de interacción familiar, que se encuentran mediadas por la expresión de afectos, sentimientos y emociones de sus miembros.

Otro aspecto que interesa en la selección de un instrumento es la validez de contexto. A este respecto, con el CTF se tuvo en cuenta el hecho de haberse desarrollado para el contexto de América Latina y Caribe (Álvarez-González y Zabaleta, 2020). Precisamente, Extremera y Fernández-Berrocal (2005) señalan que la mayor parte de instrumentos para evaluar la inteligencia emocional y las competencias emocionales, se han desarrollado en el ámbito anglosajón, y por ello, consideran necesario que este tipo de cuestionarios refleje la idiosincrasia de cada cultural.

Tabla 3.

Estructura y variables de las escalas de CTF seleccionadas

ESTRUCTURA Y VARIABLES DE ESTUDIO. Cuestionario Cómo es Tu Familia, CTF (adolescentes)

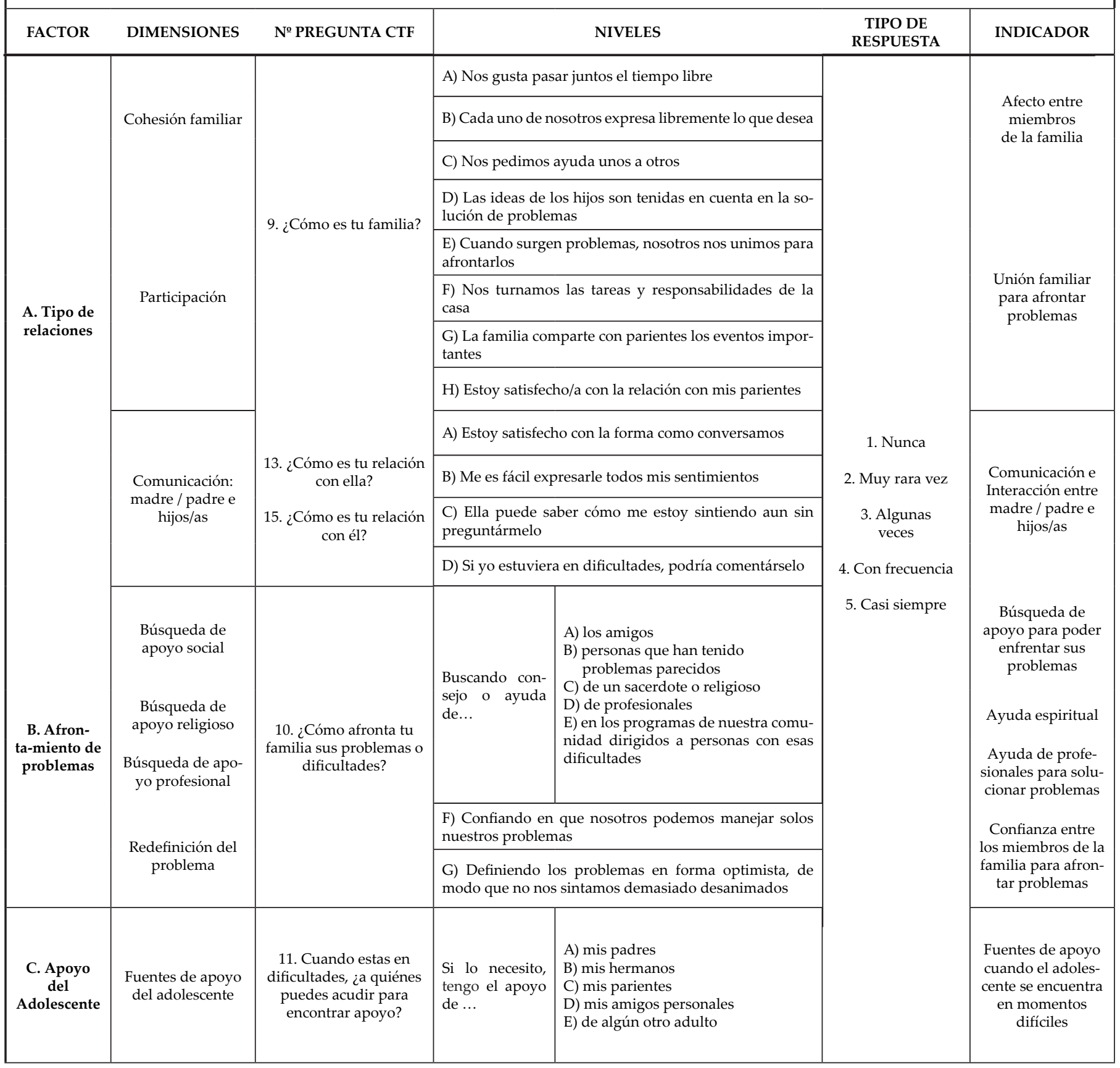


En cuanto al constructo, los autores del CTF utilizado en esta investigación informaron de su elaboración y adaptación a partir de otros precedentes, principalmente: FACES III de Olson et al., del que se adaptaron, principalmente, los ítems relativos a Cohesión familiar y adaptabilidad (Toma de decisiones); McCubbin et al. (1982), del que se adaptaron ítems de Comunicación familiar; y FILE de McCubbin, Patterson et al. (1981), del que se adaptaron ítems de Fuentes de estrés del último año.

En cuanto al protocolo que se estableció para abordar los objetivos, a partir del cuestionario CTF, se seleccionaron algunas escalas del factor 1 (Tipo de relaciones), y todas las escalas que componen los factores 2 (Afrontamiento de problemas) y 3 (Fuentes de apoyo de/la adolescente) (tabla_4). En este estudio se entiende por escala la selección de un enunciado que agrupa una serie de preguntas, y se asume este concepto siguiendo la definición de Bolaños-García y González-Ruiz (2012, p.717), que indican que es el conjunto de declaraciones que miden el grado en que las personas están de acuerdo o en desacuerdo.

Factor 1 Tipos de relaciones. Se han tomado las preguntas 9, 13 y 15 completas del CTF. La pregunta 9 contiene la Escala A: Cohe- sión familiar. La razón de esta denominación a la Escala A es que los ítems que la componen aluden, en conjunto, a la unión familiar expresada en afectos, afrontamiento y participación en los problemas familiares. Las preguntas 13 y 15 contienen las escalas D y E, que aluden a la relación y comunicación con la madre y el padre respectivamente.

Factor 2 Afrontamiento de problemas. Se ha tomado la pregunta 11 completa del CTF, y se denomina: Escala B: Apoyos dentro y fuera de la familia, ya que los ítems que la componen abarcan los apoyos de los distintos miembros de la familia, así como el de personas externas al grupo familiar

Factor 3 Fuentes de apoyo del/a adolescente. Se ha tomado la pregunta 11 completa del CTF, y se denomina: Escala C: Apoyos formales e informales ante problemas familiares, ya que los ítems que la componen enumeran las posibilidades de ayuda/consulta, formal e informal, a los que la familia puede acudir antes problemas o dificultades.

La estructura de factores y escalas seleccionados para el estudio se detallan en la tabla 4.

Tabla 4.

Estructura del cuestionario: Cómo es TU familia y escalas utilizadas en la investigación

Estructura de factores del Cuestionario Cómo es TU familia (CTF)

\begin{tabular}{|c|c|c|}
\hline Factores & Escalas & Escalas de estudio \\
\hline \multirow{9}{*}{$\begin{array}{l}\text { Factor } 1 \\
\text { Tipo de relaciones }\end{array}$} & a) Tipo de familia & \multirow{9}{*}{$\begin{array}{l}\text { En cursiva, ítems: e, f, g } \\
\text { (tablas } 4,5 \text { y } 6 \text { ) }\end{array}$} \\
\hline & b) № de miembros & \\
\hline & c) Etapa ciclo vital & \\
\hline & d) Jerarquía y límites & \\
\hline & e) Cohesión familiar & \\
\hline & f) Participación & \\
\hline & g) Comunicación progenitores/hijos & \\
\hline & h) Comunicación pareja & \\
\hline & i) Rutinas familiares & \\
\hline \multirow{4}{*}{$\begin{array}{l}\text { Factor } 2 \\
\text { Afrontamiento de pro- } \\
\text { blemas }\end{array}$} & a) Búsqueda apoyo social & \multirow{4}{*}{$\begin{array}{l}\text { Escala Afrontamiento de proble- } \\
\text { mas completa }\end{array}$} \\
\hline & b) Búsqueda apoyo religioso & \\
\hline & c) Búsqueda apoyo profesional & \\
\hline & d) Redefinición del problema (RP) & \\
\hline \multirow{5}{*}{$\begin{array}{l}\text { Factor } 3 \\
\text { Fuentes de apoyo del } \\
\text { adolescente }\end{array}$} & a) Si lo necesito, tengo el apoyo de mis padres & \multirow{5}{*}{$\begin{array}{l}\text { Escala Fuentes de apoyo del } \\
\text { adolescente completa }\end{array}$} \\
\hline & b) Si lo necesito, tengo el apoyo de mis hermanos & \\
\hline & c) Si lo necesito, tengo el apoyo de mis parientes & \\
\hline & d) Si lo necesito, tengo el apoyo de mis amigos personales & \\
\hline & e) Si lo necesito, tengo el apoyo de algún otro adulto & \\
\hline
\end{tabular}

(Elaboración propia. En cursiva las escalas utilizadas en la investigación)

\section{Análisis de datos}

La información del CTF se ha analizado mediante SPSS (V.25 IBM SPSS Statistics v 25.0 Windows, USA). Para analizar los datos de esta investigación, y para los dos primeros objetivos específicos se han aplicado las pruebas $t$ de Student para muestras independientes, con respecto a las edades de los/as adolescentes (tabla_6), y en relación al sexo (tabla_7), y ANOVA 1 factor de efectos fijos (ANOVA 1 f.e.f.) para el tercer objetivo específico, que contempla los grupos por sexo y edad (tabla_8). Por un lado, se optó por la t de Student porque interesa comprobar si se dan diferencias sig- nificativas entre los grupos de estudio con respecto a las variables estudiadas, y tal y como se constata en investigaciones previas, para ello se toman las puntuaciones de la escala Likert, que van de 1 nunca a 5 casi siempre (tabla_3), y se han calculado las medias para cada ítem. Siguiendo el mismo criterio, y con respecto al tercer objetivo, se aplicó una prueba ANOVA 1 (f.e.f.), ya que es la adecuada a la naturaleza de las variables sexo y edad.

Los valores de fiabilidad de los que informa el Instrumento Cómo es TU familia, se obtuvieron, según sus autores, en el estudio piloto del mismo, y los correspondientes a las escalas seleccionadas en esta investigación son: 
Tabla 5.

$\alpha$ de Cronbach. Fiabilidad en escalas Cómo es Tu familia

\begin{tabular}{lc}
\hline ESCALAS & a de Cronbach \\
\hline A_COHESIÓN_FAMILIAR & .79 \\
B_PARTICIPACIÓN_SOLUCIÓN_PROBLEMAS & .79 \\
C_AFRONTAMIENTO_PROBLEMAS & $.56 / .62 / .62 / .62$ \\
$\left.{ }^{*}\right)$ 4 formas: Apoyo social / profesional / religioso / redefinición del problema) & .82 \\
D_RELACION_CON_LA_MADRE & .78 \\
E_RELACION_CON_EL_PADRE & \\
\hline
\end{tabular}

${ }^{*}$ ) Los valores $\alpha$ corresponden a las cuatro formas. Elaboración propia

Con respecto a la forma para los progenitores (Cómo es $\mathrm{Su}$ Familia, CSF), aunque no se utilizó en este estudio, se aportan los valores ( $\alpha$ de Cronbach) de las escalas paralelas a las del CTF, y son: Cohesión familiar (.76); Participación en la solución de problemas (.76); Comunicación con el padre (.81); Comunicación con la madre (.79), y en Afrontamiento de problemas $(.53$ / .61 / $.61 / .61$, respectivamente).

\section{Resultados}

Los resultados encontrados en el presente estudio muestran que existen diferencias de género, en los dos tramos de edad, entre las puntuaciones de las variables estudiadas.

\section{Contraste por edades}

Las diferencias por edad evidencian resultados coherentes con el perfil de la muestra, a priori, ni los/as adolescentes ni sus familias presentan problemáticas específicas. En la Escala A (Cohesión familiar) (tabla_6) todos los ítems muestran medias elevadas, por encima de 3.5 , es decir, consideran que todos los indicadores de cohesión se dan en sus familias -con frecuencia-, y esta percepción se da en valores muy semejantes en ambos rangos de edad, por lo que no aparecen diferencias significativas, salvo en la -satisfacción- con la relación con parientes- a favor de los más jóvenes.

En la Escala B (Participación en la solución de problemas) el ítem más valorado es -el apoyo de los padres- y es significativamente más alto por parte de los más jóvenes, lo que parece indicar la progresiva autonomía que van adquiriendo. Los otros dos resultados significativos se refieren al apoyo de un -amigo personal-, a favor del grupo mayor, lo que también es esperable en la etapa de la adolescencia. En cuanto al apoyo de -otro adulto-, también es significativo, pero es la puntuación más baja de la escala, lo que denota que no es un recurso frecuente ante los problemas.

Los resultados de la escala C (Afrontamiento de problemas), aparecen diferenciados claramente en dos bloques de ítems. Por un lado, las puntuaciones correspondientes a la ayuda -externa-, amigos, profesionales, etc. y por otro, la ayuda procedente del propio núcleo familiar. En el primer caso, las puntuaciones apenas alcanzan el promedio (2.5 poca frecuencia), y se recurre más al recurso de -personas con problemas semejantes-. En el segundo, la ayuda familiar se valora más, aunque se queda justo en el promedio.

En las escalas D (Relación con la madre) y E (Relación con el padre) no hay diferencias significativas en los dos grupos de edad, y el ítem mejor valorado en ambos grupos es la -comunicación con la madre-; los restantes ítems de esta escala también obtienen puntuaciones medias-altas, y ligeramente superiores en el grupo más joven. Respecto a la -relación con el padre- todas las puntuaciones son ligeramente inferiores a las de la madre, especialmente en el grupo de más edad y con respecto a su capacidad para facilitar la expresión emocional (ítems 2 y 3).

\section{Contraste por sexo}

En Cohesión_familiar, como se observa en la tabla_7, los ocho ítems obtienen puntuaciones ligeramente por encima de la media, y solo un caso muestra diferencia significativa, a favor de las adolescentes: compartir eventos con familiares.

En Participación_solución_problemas, las puntuaciones muestran que ambos sexos valoran el apoyo (las medias superan el promedio) sobre todo, de los padres y resto de familiares. El único valor significativo es la valoración del apoyo de un amigo personal, y como resulta esperable en la adolescencia, las puntuaciones son altas en ambos casos, pero algo más en el caso de las adolescentes.

Al igual que sucede en la tabla_6 (diferencias por edad) en la tabla_7 también se aprecia que los resultados de Afrontamiento_ problemas, aparecen en dos bloques. Las puntuaciones correspondientes a la ayuda -externa-, apenas alcanzan el promedio (2.5 poca frecuencia). En cuanto a la ayuda procedente del propio núcleo familiar obtiene puntuaciones algo más altas, siendo la más elevada la búsqueda de nuevas formas de afrontamiento. En ningún caso se dan diferencias significativas. Hay que señalar que los ítems 6 y 7 corresponden a lo que los autores del CTF consideran como el subfactor Redefinición del problema. Y aunque los valores en estos dos ítems no son significativos, los adolescentes varones muestran una mayor confianza en sí mismos para resolver los problemas, mientras que ellas se perciben con mayor optimismo para no caer en el desánimo.

Las escalas de Relación con la madre/padre, siguen la misma tendencia que en las diferencias por edad. Los y las adolescentes, en general, valoran con puntuaciones ligeramente más altas la relación con la madre, y en esta línea, quienes valoran más la relación con el padre y con la madre son los adolescentes varones. En la relación con el padre, las adolescentes otorgan las puntuaciones más bajas en los cuatro ítems, y estas son significativas.

A la vista de los resultados específicos para cada una de las variables estudiadas, se planteó el ANOVA. Con las variables de sexo y edad se establecieron cuatro grupos de tamaño similar, para contrastar las diferencias de medias (tabla_8). 
Tabla 6.

Análisis inferencial (t de Student). Diferencias por edad.

\begin{tabular}{|c|c|c|c|c|}
\hline \multirow[b]{2}{*}{ ESCALAS E ÍTEMS } & \multicolumn{2}{|c|}{ Media (Desv.Est.) } & \multicolumn{2}{|c|}{ Test Student } \\
\hline & $\begin{array}{c}12-14 \\
(n=430)\end{array}$ & $\begin{array}{c}15-17 \\
(n=491)\end{array}$ & Valor & P-valor \\
\hline \multicolumn{5}{|l|}{ A_COHESIÓN_FAMILIAR } \\
\hline Agrado por pasar tiempo libre juntos & $3.81(1.13)$ & $3.75(1.13)$ & 0.82 NS & .412 \\
\hline Expresión fácil de lo que se desea & $3.51(1.14)$ & $3.54(1.12)$ & $-0.35^{N S}$ & .753 \\
\hline Pedir ayuda unos a otros & $4.03(1.08)$ & $3.90(1.13)$ & $1.70^{N S}$ & .090 \\
\hline Ideas de los hijos son tenidas en cuenta & $3.54(1.24)$ & $3.48(1.23)$ & $0.77^{N S}$ & .441 \\
\hline Unidos para afrontar los problemas & $3.64(1.27)$ & $3.77(1.20)$ & $-1.54^{N S}$ & .124 \\
\hline Turnos en tareas y responsabilidades de la casa & $3.82(1.19)$ & $3.71(1.22)$ & $1.33^{N S}$ & .183 \\
\hline Compartir eventos con parientes cercanos & $4.20(1.10)$ & $4.16(1.04)$ & 0.62 NS & .535 \\
\hline Satisfacción con la relación con los parientes & $4.24(1.04)$ & $4.01(1.14)$ & $3.22^{* *}$ & .001 \\
\hline \multicolumn{5}{|l|}{ B_PARTICIPACIÓN_SOLUCIÓN_PROBLEMAS } \\
\hline Apoyo de los padres & $4.23(1.04)$ & $4.06(1.16)$ & $2.23 *$ & .026 \\
\hline Apoyo de los hermanos & $3.43(1.38)$ & $3.54(1.37)$ & $-1.15^{N S}$ & .251 \\
\hline Apoyo de algún pariente & $3.17(1.30)$ & $3.10(1.31)$ & $0.79^{N S}$ & .430 \\
\hline Apoyo de algún amigo personal & $3.31(1.28)$ & $3.66(1.23)$ & $-4.13^{* *}$ & .000 \\
\hline Apoyo de otro adulto & $2.24(1.33)$ & $2.51(1.34)$ & $-3.03^{* *}$ & .003 \\
\hline \multicolumn{5}{|l|}{ C_AFRONTAMIENTO_PROBLEMAS } \\
\hline Consejo o ayuda de amigos & $2.70(1.24)$ & $2.55(1.14)$ & $1.91^{\mathrm{NS}}$ & .056 \\
\hline Consejolayuda en personas con problemas parecidos & $2.67(1.24)$ & $2.47(1.08)$ & $2.56^{* *}$ & .011 \\
\hline Consejo o ayuda en sacerdote, religioso, ... & $2.43(1.31)$ & $2.26(1.30)$ & $1.99 *$ & .047 \\
\hline Consejo o ayuda de profesionales & $2.20(1.27)$ & $2.21(1.23)$ & 0.09 NS & .927 \\
\hline Con los programas existentes en la Comunidad & 1.85 (1.08) & $1.63(0.91)$ & $3.30^{* *}$ & .001 \\
\hline Confianza en que ellos solos pueden afrontar y manejar los problemas & $3.00(1.36)$ & $3.21(1.27)$ & $2.30 *$ & .022 \\
\hline Siendo optimistas para no caer en el desánimo & $3.02(1.36)$ & $3.26(1.26)$ & $2.68^{* *}$ & .008 \\
\hline Con búsqueda de nuevas formas de afrontamiento & $3.82(1.17)$ & $3.84(1.15)$ & $-0.23^{N S}$ & .821 \\
\hline \multicolumn{5}{|l|}{ D_RELACION_CON_LA_MADRE } \\
\hline Satisfacción con la forma en que se conversa & 4.28 (1.09) & $4.30(1.10)$ & -0.23 NS & .822 \\
\hline Facilidad para expresar sus sentimientos & $3.73(1.27)$ & $3.64(1.25)$ & $1.05^{N S}$ & .292 \\
\hline Ella sabe mis sentimiento aun sin preguntarme & $4.06(1.21)$ & $3.90(1.27)$ & 1.92 NS & .055 \\
\hline En dificultades, puede contar con ella & $3.93(1.30)$ & $3.91(1.25)$ & $0.24^{\text {NS }}$ & .814 \\
\hline \multicolumn{5}{|l|}{ E_RELACION_CON_EL_PADRE } \\
\hline Satisfacción con la forma en que se conversa & $3.73(1.38)$ & $3.68(1.35)$ & $0.57^{\mathrm{NS}}$ & .569 \\
\hline Facilidad para expresar sus sentimientos & $3.15(1.41)$ & $2.97(1.35)$ & $1.96^{\mathrm{NS}}$ & .050 \\
\hline Él sabe mis sentimiento aun sin preguntarme & $3.12(1.46)$ & $2.95(1.49)$ & $1.67^{N S}$ & .096 \\
\hline En dificultades, puede contar con él & $3.31(1.48)$ & $3.28(1.51)$ & $0.26^{N S}$ & .795 \\
\hline
\end{tabular}

\footnotetext{
N.S. = NO significativo $\quad *$ = Significativo $\quad * *=$ Altamente significativo
} 
Tabla 7.

Análisis inferencial. $t$ de Student. Diferencias por sexo.

\begin{tabular}{|c|c|c|c|c|}
\hline \multirow[b]{2}{*}{ ESCALAS E ÍTEMS } & \multicolumn{2}{|c|}{ Media (Desv.Est.) } & \multicolumn{2}{|c|}{ Test Student } \\
\hline & $\begin{array}{c}\text { Hombres } \\
(\mathrm{n}=482)\end{array}$ & $\begin{array}{r}\text { Mujeres } \\
(\mathrm{n}=439)\end{array}$ & Valor & P-valor \\
\hline \multicolumn{5}{|l|}{ A_COHESIÓN_FAMILIAR } \\
\hline Agrado por pasar tiempo libre juntos & $3.75(1.09)$ & $3.81(1.16)$ & $-0.81^{\mathrm{NS}}$ & .417 \\
\hline Expresión fácil de lo que se desea & $3.58(1.08)$ & $3.47(1.18)$ & $1.46^{\mathrm{NS}}$ & .146 \\
\hline Pedir ayuda unos a otros & $3.98(1.07)$ & $3.94(1.15)$ & 0.61 NS & .540 \\
\hline Ideas de los hijos son tenidas en cuenta & $3.53(1.22)$ & $3.48(1.24)$ & 0.53 NS & .595 \\
\hline Unidos para afrontar los problemas & $3.71(1.23)$ & $3.71(1.25)$ & -0.01 NS & .991 \\
\hline Turnos en tareas y responsabilidades de la casa & $3.72(1.20)$ & $3.81(1.21)$ & $-1.05^{\mathrm{NS}}$ & .295 \\
\hline Compartir eventos con parientes cercanos & $4.09(1.09)$ & $4.28(1.04)$ & $-2.70^{* *}$ & .007 \\
\hline Satisfacción con la relación con los parientes & $4.15(1.12)$ & $4.08(1.07)$ & 0.96 NS & .335 \\
\hline \multicolumn{5}{|l|}{ B_PARTICIPACIÓN_SOLUCIÓN_PROBLEMAS } \\
\hline Apoyo de los padres & $4.13(1.10)$ & $4.15(1.11)$ & $-0.23^{N S}$ & .816 \\
\hline Apoyo de los hermanos & $3.48(1.35)$ & $3.50(1.40)$ & $-0.29^{N S}$ & .770 \\
\hline Apoyo de algún pariente & $3.15(1.29)$ & $3.12(1.32)$ & 0.34 NS & .773 \\
\hline Apoyo de algún amigo personal & $3.37(1.30)$ & $3.64(1.20)$ & $-3.21^{* *}$ & .001 \\
\hline Apoyo de otro adulto & $2.39(1.33)$ & $2.38(1.35)$ & $0.08^{\mathrm{NS}}$ & .938 \\
\hline \multicolumn{5}{|l|}{ C_AFRONTAMIENTO_PROBLEMAS } \\
\hline Consejo o ayuda de amigos & $2.61(1.20)$ & $2.62(1.17)$ & $-0.05^{\mathrm{NS}}$ & .961 \\
\hline Consejolayuda en personas con problemas parecidos & $2.63(1.18)$ & $2.49(1.14)$ & $1.86^{\mathrm{NS}}$ & .063 \\
\hline Consejo o ayuda en sacerdote, religioso, ... & $2.29(1.30)$ & $2.39(1.31)$ & -1.08 NS & .280 \\
\hline Consejo o ayuda de profesionales & $2.18(1.22)$ & $2.24(1.28)$ & $-0.78^{N S}$ & .438 \\
\hline Con los programas existentes en la Comunidad & $1.75(1.03)$ & $1.70(0.96)$ & $0.83^{\mathrm{NS}}$ & .404 \\
\hline Confianza en que ellos solos pueden afrontar y manejar los problemas & $3.19(1.33)$ & $3.04(1.30)$ & 1.68 NS & .093 \\
\hline Siendo optimistas para no caer en el desánimo & $3.07(1.31)$ & $3.24(1.30)$ & -1.93 NS & .054 \\
\hline Con búsqueda de nuevas formas de afrontamiento & $3.78(1.19)$ & $3.89(1.13)$ & $-1.47^{\mathrm{NS}}$ & .142 \\
\hline \multicolumn{5}{|l|}{ D_RELACION_CON_LA_MADRE } \\
\hline Satisfacción con la forma en que se conversa & $4.30(1.08)$ & $4.27(1.12)$ & $0.45^{\mathrm{NS}}$ & .650 \\
\hline Facilidad para expresar sus sentimientos & $3.75(1.26)$ & $3.62(1.27)$ & 1.54 NS & .124 \\
\hline Ella sabe mis sentimiento aun sin preguntarme & $4.00(1.20)$ & $3.95(1.30)$ & 0.69 NS & .488 \\
\hline En dificultades, puede contar con ella & $3.91(1.25)$ & $3.93(1.30)$ & $-0.19^{\mathrm{NS}}$ & .852 \\
\hline \multicolumn{5}{|l|}{$E \_R E L A C I O N \_C O N \_E L \_P A D R E$} \\
\hline Satisfacción con la forma en que se conversa & $3.85(1.30)$ & $3.54(1.41)$ & $3.40^{* *}$ & .001 \\
\hline Facilidad para expresar sus sentimientos & $3.21(1.40)$ & $2.88(1.33)$ & $3.53^{* *}$ & .000 \\
\hline Él sabe mis sentimiento aun sin preguntarme & $3.17(1.45)$ & $2.88(1.49)$ & $2.94^{* *}$ & .003 \\
\hline En dificultades, puede contar con él & $3.41(1.47)$ & $3.17(1.51)$ & $2.38 *$ & .018 \\
\hline
\end{tabular}

El objetivo de realizar el análisis ANOVA es lograr una idea más clara sobre la conducta de los y las adolescentes en la búsqueda de ayuda ante los problemas familiares, así como su percepción sobre el clima de comunicación y relación con los padres. En este sentido, parece que la edad marca una diferencia, por ejemplo, en Cohesión_familiar, en los dos últimos ítems satisfacción con la relación con parientes (familia extensa) se da una disminución de los valores entre los y las adolescentes mayores (15-17), y esta menor valoración es significativa, sobre todo en las mujeres.
Las escalas B y C deben analizarse conjuntamente, la B (Participación_solución_problemas) se centra en los apoyos procedentes del propio grupo familiar, así como de personas próximas a dicho núcleo. La escala C (Afrontamiento_problemas), salvo los tres últimos ítems, los restantes se centran en el "apoyo social", (consejo o ayuda de personas externas a la familia). A este respecto, tanto en los resultados por sexo o edad (tablas 6 y 7), como en el ANOVA (tabla_8), se observa una preferencia por abordar los problemas desde el propio grupo familiar, antes que acudir a recursos externos, ya sean profesionales o personas con proble- 
Tabla 8.

Análisis inferencial. ANOVA Diferencias por Sexo y Edad.

\begin{tabular}{|c|c|c|c|c|c|c|}
\hline & \multicolumn{4}{|c|}{ Media (Desviación estándar) } & \multicolumn{2}{|c|}{ ANOVA 1 f.e.f. } \\
\hline & $\begin{array}{c}\text { Hombre } \\
12-14 \\
(\mathrm{n}=222)\end{array}$ & $\begin{array}{c}\text { Hombre } \\
15-17 \\
(\mathrm{n}=260)\end{array}$ & $\begin{array}{c}\text { Mujer } \\
12-14 \\
(n=208)\end{array}$ & $\begin{array}{c}\text { Mujer } \\
\text { 15-17 } \\
(\mathrm{n}=231)\end{array}$ & Valor & P-valor \\
\hline \multicolumn{7}{|l|}{ A_COHESIÓN_FAMILIAR } \\
\hline Agrado por pasar tiempo libre juntos & $3.86(1.05)$ & $3.66(1.12)$ & $3.76(1.20)$ & $3.86(1.13)$ & $1.81^{\mathrm{NS}}$ & .144 \\
\hline Expresión fácil de lo que se desea & $3.55(1.07)$ & $3.60(1.09)$ & $3.48(1.20)$ & $3.46(1.16)$ & $0.81^{\mathrm{NS}}$ & .490 \\
\hline Pedir ayuda unos a otros & $4.12(1.03)$ & $3.87(1.08)$ & $3.94(1.12)$ & $3.94(1.18)$ & $2.044^{\mathrm{NS}}$ & .107 \\
\hline Ideas de los hijos son tenidas en cuenta & $3.59(1.23)$ & $3.48(1.22)$ & $3.49(1.25)$ & $3.48(1.23)$ & $0.43^{\mathrm{NS}}$ & .731 \\
\hline Unidos para afrontar los problemas & $3.68(1.25)$ & $3.74(1.21)$ & $3.60(1.29)$ & $3.81(1.20)$ & $1.05^{\mathrm{NS}}$ & .370 \\
\hline Turnos en tareas y responsabilidades de la casa & $3.87(1.12)$ & $3.60(1.26)$ & $3.77(1.26)$ & $3.84(1.16)$ & $2.49^{\mathrm{NS}}$ & .059 \\
\hline Compartir eventos con parientes cercanos & $4.08(1.14)$ & $4.09(1.04)$ & $4.33(1.05)$ & $4.23(1.03)$ & $2.75 *$ & .041 \\
\hline Satisfacción con la relación con los parientes & $4.21(1.09)$ & $4.10(1.14)$ & $4.27(0.97)$ & $3.90(1.13)$ & $4.84^{* *}$ & .002 \\
\hline \multicolumn{7}{|l|}{ B_PARTICIPACIÓN_SOLUCIÓN_PROBLEMAS } \\
\hline Apoyo de los padres & $4.21(1.02)$ & $4.07(1.15)$ & $4.25(1.06)$ & $4.06(1.16)$ & $1.69^{\mathrm{NS}}$ & .167 \\
\hline Apoyo de los hermanos & $3.48(1.30)$ & $3.47(1.40)$ & $3.38(1.45)$ & $3.61(1.34)$ & $1.06^{\mathrm{NS}}$ & .367 \\
\hline Apoyo de algún pariente & $3.18(1.28)$ & $3.12(1.30)$ & $3.16(1.32)$ & $3.08(1.31)$ & $0.25^{\mathrm{NS}}$ & .861 \\
\hline Apoyo de algún amigo personal & $3.13(1.32)$ & $3.57(1.26)$ & $3.50(1.21)$ & $3.76(1.18)$ & $9.83^{* *}$ & .000 \\
\hline Apoyo de otro adulto & $2.26(1.36)$ & $2.49(1.31)$ & $2.22(1.31)$ & $2.53(1.38)$ & $3.12 *$ & .025 \\
\hline \multicolumn{7}{|l|}{ C_AFRONTAMIENTO_PROBLEMAS } \\
\hline Consejo o ayuda de amigos & $2.69(1.26)$ & $2.55(1.15)$ & $2.71(1.22)$ & $2.54(1.13)$ & $1.23^{\mathrm{NS}}$ & .298 \\
\hline Consejolayuda en personas con problemas parecidos & $2.69(1.26)$ & $2.59(1.10)$ & $2.66(1.21)$ & $2.34(1.05)$ & $4.06^{* *}$ & .007 \\
\hline Consejo o ayuda en sacerdote, religioso, ... & $2.45(1.32)$ & $2.16(1.27)$ & $2.41(1.31)$ & $2.37(1.32)$ & $2.35^{\mathrm{NS}}$ & .071 \\
\hline Consejo o ayuda de profesionales & $2.23(1.28)$ & $2.14(1.17)$ & $2.18(1.27)$ & $2.30(1.29)$ & $0.70^{\mathrm{NS}}$ & .554 \\
\hline Con los programas existentes en la Comunidad & $1.92(1.11)$ & $1.62(0.94)$ & $1.77(1.04)$ & $1.63(0.89)$ & $4.35^{* *}$ & .005 \\
\hline $\begin{array}{l}\text { Confianza en que ellos solos pueden afrontar y manejar } \\
\text { los problemas }\end{array}$ & $3.14(1.36)$ & $3.22(1.29)$ & $2.86(1.34)$ & $3.19(1.24)$ & $3.40 *$ & .017 \\
\hline Siendo optimistas para no caer en el desánimo & $2.99(1.35)$ & $3.14(1.28)$ & $3.06(1.37)$ & $3.40(1.23)$ & $4.09^{* *}$ & .007 \\
\hline Con búsqueda de nuevas formas de afrontamiento & $3.87(1.18)$ & $3.69(1.19)$ & $3.77(1.16)$ & $4.00(1.09)$ & $3.05 *$ & .028 \\
\hline \multicolumn{7}{|l|}{ D_RELACION_CON_LA_MADRE } \\
\hline Satisfacción con la forma en que se conversa & $4.29(1.08)$ & $4.32(1.07)$ & $4.27(1.10)$ & $4.27(1.14)$ & $0.11^{\mathrm{NS}}$ & .957 \\
\hline Facilidad para expresar sus sentimientos & $3.88(1.24)$ & $3.64(1.26)$ & $3.58(1.29)$ & $3.65(1.25)$ & $2.32^{\mathrm{NS}}$ & .074 \\
\hline Ella sabe mis sentimiento aun sin preguntarme & $4.11(1.15)$ & $4.92(1.23)$ & $4.01(1.27)$ & $3.88(1.32)$ & $1.46^{\mathrm{NS}}$ & .226 \\
\hline En dificultades, puede contar con ella & $3.98(1.25)$ & $3.85(1.26)$ & $3.88(1.34)$ & $3.97(1.25)$ & $0.55^{\mathrm{NS}}$ & .647 \\
\hline \multicolumn{7}{|l|}{$E \_R E L A C I O N_{-} C O N_{-} E L \_P A D R E$} \\
\hline Satisfacción con la forma en que se conversa & $3.96(1.26)$ & $3.76(1.33)$ & $3.49(1.45)$ & $3.58(1.37)$ & $4.75^{* *}$ & .003 \\
\hline Facilidad para expresar sus sentimientos & $3.36(1.42)$ & $3.08(1.38)$ & $2.93(1.37)$ & $2.83(1.30)$ & $5.92^{* *}$ & .001 \\
\hline Él sabe mis sentimiento aun sin preguntarme & $3.33(1.43)$ & $3.04(1.46)$ & $2.90(1.46)$ & $2.86(1.52)$ & $4.41^{* *}$ & .004 \\
\hline En dificultades, puede contar con él & $3.48(1.46)$ & $3.35(1.49)$ & $3.13(1.50)$ & $3.20(1.53)$ & $2.20^{\mathrm{NS}}$ & .086 \\
\hline
\end{tabular}

N.S. $=$ NO significativo $\quad *$ Significativo $\quad * *=$ Altamente significativo

mas semejantes. Además, esta tendencia se acentúa en el grupo de los y las adolescentes mayores.

La influencia de la edad también está presente en la relación que los y las adolescentes mantienen con el padre y la madre. Sobre todo, en el caso de la relación con el padre se aprecia un descenso generalizado y significativo en la satisfacción con la misma, así como en la facilidad para establecer comunicación con él. En cuanto a la relación con la madre, los resultados son más heterogéneos, y aunque también se observan descensos en algunos ítems, estas diferencias no llegan a ser significativas en ningún caso.

\section{Discusión y conclusiones}

Con respecto a las diferencias por edad en estudios sobre IE y sobre competencias emocionales en la familia, desde un enfoque cognitivo (Extremera y Fernández-Berrocal, 2004) parece que sucede lo mismo que con las capacidades intelectuales, que estás se 
incrementan a medida que la persona madura. Esto tiene implicaciones, sobre todo, con respecto a los mejores momentos para intervenir desde la educación emocional (Garaigordobil, 2018).

En esta investigación, respecto a la percepción que los y las adolescentes manifiestan sobre la comunicación con sus padres y madres, no se han encontrado diferencias significativas por sexo cuando se trata de los aspectos de comunicación con la madre, y este resultado es coherente con el estudio de Polo del Río et al. (2012) en el que también informan de la ausencia de diferencias entre sexos en cuanto a la comunicación y afecto que perciben de sus padres. En este caso, los resultados tampoco fueron significativos en el caso del padre.

En cuanto a las conclusiones con respecto a los objetivos planteados, el primer objetivo, centrado en el análisis de las percepciones de los y las adolescentes de la muestra, con respecto a su interacción y participación en la familia, los resultados obtenidos en función del sexo y la edad y la edad de la muestra de adolescentes participantes reflejan que las puntuaciones más elevadas se obtienen principalmente en las subescalas de a) relación con la madre, b) cohesión familiar, c) participación en la solución de problemas y d) consulta y búsqueda de apoyos.

En la Relación con la madre, sin embargo, no se han dado diferencias significativas entre sexos, y sí consenso en las puntuaciones otorgadas por hombres y mujeres. De esta forma, se señala a la figura de la madre como un elemento clave en el núcleo familiar.

Por último, hay que señalar las bajas puntuaciones que obtiene la muestra de adolescentes en todos los ítems referidos a la búsqueda de ayuda fuera de la familia, siendo la puntuación más baja la referida a los posibles programas de apoyo de la comunidad. A este respecto, este resultado puede indicar la escasez de recursos que presenta la ciudad de Loja en este sentido, y por lo tanto, la conveniencia de continuar con estudios que tengan en cuenta las necesidades de familias y adolescentes con respecto a las variables analizadas en esta investigación.

Con respecto al segundo objetivo, identificar resultados en las cinco escalas estudiadas con relevancia para la educación emocional, en relación a la muestra estudiada, cabe destacar aspectos en cada una de las cinco escalas. En la Cohesión familiar, se asume que los ítems incluidos constituyen indicadores de un clima familiar adecuado para desarrollar en hijos e hijas competencias emocionales, por lo que puede ayudar a identificar necesidades en este ámbito.

En las dos siguientes: Participación en solución problemas y Afrontamiento de problemas, en la muestra, se evidencia una mayor valoración de los recursos propios de la familia (hermanos, padres, parientes) frente a los apoyos procedentes del exterior, y a pesar de que se valora positivamente la búsqueda de formas innovadoras de afrontamiento, posiblemente, y en el caso concreto de esta muestra, esta percepción podría llevarles a plantear cierta resistencia hacia su implicación en un programa de intervención en competencias emocionales, precisamente por valorar los propios recursos familiares por delante de otros apoyos externos. Este planteamiento se ha puesto de manifiesto al comprobar que el recurso peor valorado son los programas existentes en la Comunidad.

Por último, en las escalas de Relación con la madre y el padre, los resultados son interesantes, ya que la valoración de la madre en situaciones emocionales (expresión y comprensión de los sentimientos, comunicación ...) es prácticamente unánime para ambos sexos y en todas las edades, pero no sucede igual con la percepción sobre el padre; los hijos varones valoran bien su relación con él, aunque algo menos que con la madres, y las hijas otorgan valoraciones más bajas y significativas, situación que señala la necesidad de mejora en este aspecto.

\section{Implicaciones para la educación emocional}

Implicación del instrumento. En el marco de las edades que abarca el cuestionario CTF, este podría contribuir en los estudios de pre y postest en los que se trata de evaluar los desarrollos logrados a través de programas de educación emocional. Por lo general, cuando se evalúa la eficacia de estos programas, las pruebas pre y postest se dirigen a la misma población que ha recibido la formación en educación emocional. Y dado que estos desarrollos se producen en un medio (familiar) caracterizado por el carácter sistémico de sus relaciones, puede ser adecuado que la evaluación del efecto de estos programas sea igualmente sistémica. A este respecto, una evaluación sistémica debe contemplar la recogida de información de una forma articulada entre participantes, y en este caso, entre integrantes del sistema familiar, para recoger información no solo de las percepciones individuales, sino también de las interacciones. En consecuencia, la evaluación sistémica requiere del diseño de herramientas adaptadas a este fin, como es el caso de los cuestionarios del Manual de indicadores de salud. Familia y adolescencia (Hernández et al. 1996) utilizado en este estudio.

Sobre el valor de este instrumento para triangular las percepciones de padres e hijos/as sobre su clima familiar, junto a las pruebas de pre y postest de una intervención en educación emocional, dirigidas a los padres, podrían sumarse las escalas del CTF, para contrastar dichas percepciones de mejora entre los miembros de la familia.

En investigaciones como esta, dónde es preciso indagar en relaciones y de comunicación interpersonales, en contextos determinados, como la familia, o incluso, percepciones intrapersonales, los datos recabados suelen ser autoinformados, y aunque se reconoce el valor de estos instrumentos para estudiar este tipo de variables, es prácticamente inevitable que dichos datos contengan fuentes potenciales de sesgo. En esta investigación, a pesar del carácter anónimo del cuestionario, se constató, en parte de la muestra (adolescentes y familias), la reticencia a responder a determinadas cuestiones, sobre todo, si la respuesta implicaba reconocer, por ejemplo, que no se cuenta con el apoyo de los padres.

Otra limitación de este trabajo, por razones de extensión del mismo, es el haberse limitado a las percepciones de los adolescentes. El instrumento utilizado cuenta con la versión para padres, lo que permite triangular las percepciones sobre su entorno familiar.

\section{Referencias}

Álvarez-González, B. \& Zabaleta, L. I. (2020). Comunicación y gestión de problemas en el ámbito familiar de adolescentes ecuatorianos (estrategias de afrontamiento y apoyo social percibido en adolescentes ecuatorianos). En Gázquez, Molero, et al. (Comps.) Investigación en el ámbito escolar. Nuevas realidades en un acercamiento multidimensional a las variables psicológicas y educativas. 1351-1359, Dykinson. https://dialnet. unirioja.es/servlet/articulo?codigo $=7744161$

Aunola, K., Stattin, H., \& Nurmi, J. E. (2000). Parenting styles and adolescents' achievement strategies. Journal of adolescence, 23(2), 205-222. https://doi.org/10.1006/jado.2000.0308

Bar-On, R. (2000) Emotional and social intelligence: Insights from the Emotional Quotient Inventory (EQ-i). In Handbook of Emotional Intelligence: Theory, Development, Assessment and Application at Home, School and in the Workplace; Bar-On, R., Parker, J.D.A., Eds.; Jossey-Bass: USA. 363-388. https://psycnet.apa.org/record/2001-00355-018 
Bersabé, Fuentes y Motrico (2001). Análisis Psicométrico de dos escalas para evaluar estilos educativos parentales. Psicothema, 13, 678-684. https://reunido.uniovi.es/index.php/PST/article/view/7885

Bisquerra Alzina, R., \& Pérez-Escoda, N. (2007). Las competencias emocionales. Educación XX1, 10, 61-82. https:/doi. org/10.5944/educxx1.1.10.297

Bolaños-Medina, A., \& González-Ruiz, V. (2012). Deconstructing the translation of psychological tests. Meta: Journal des traducteurs/Meta: Translators' Journal, 57(3), 715-739. http://dx.doi. org/10.7202/1017088ar

Cepa Serrano, A., Heras Sevilla, D. y Fernández-Hawrylak, M. (2017). La educación emocional en la infancia: una estrategia inclusiva. Aula abierta, 46, 73-82. https://doi.org/10.17811/ rifie.46.2.2017.73-82.

Ciocanel, O., Power, K., Eriksen, A., y Gillings, K. (2017). Effectiveness of positive youth development interventions: A meta-analysis of randomized controlled trials. Journal of youth and adolescence, 46(3), 483-504. https://doi.org/10.1007/s10964-016-0555-6

Clarke, N. (2006). Emotional intelligence training: A case of caveat emptor. Human Resource Development Review, 5(4), 422-441. https://doi.org/10.1177/1534484306293844

Extremera, N., y Fernández-Berrocal, P. (2004). La inteligencia emocional: Métodos de evaluación en el aula. Revista Iberoamericana de educación, 34(1), 1-12. https://doi.org/10.35362/ rie 3412887

Extremera, N., y Fernández-Berrocal, P. (2005). La inteligencia emocional y la educación de las emociones desde el Modelo de Mayer y Salovey. Revista Interuniversitaria de Formación del Profesorado, 19(3), 63-93. http://www.redalyc.org/articulo. oa?id=27411927005

Fernández-Berrocal, P., Berrios-Martos, M. P., Extremera, N., y Augusto, J. M. (2012). Inteligencia emocional: 22 años de avances empíricos. Psicología Conductual, 20(1), 5. https://www. behavioralpsycho.com/wp-content/uploads/2019/08/01.Fernandez-Berrocal_20-1.pdf

Fuentes-Aguilar, A. P., \& Merino-Escobar, J. M. (2016). Validación de un instrumento de funcionalidad familiar. Ajayu órgano de difusión científica del departamento de psicología ucbsp, 14(2), 247-283. http://www.bibvirtual.ucb.edu.bo/ajayu/index.php/ajayu/article/view/63

Garaigordobil, M. (2018). Intrapersonal Emotional Intelligence during Adolescence: Sex Differences, Connection with other Variables, and Predictors. European Journal of Investigation in Health, Psychology and Education, 10(3), 899-914. https://doi. org/10.3390/ejihpe10030064

Gracia, E., Garcia, F., y Lila, M. (2008). Police involvement in cases of intimate partner violence against women: The influence of perceived severity and personal responsibility. Violence Against Women, 14(6), 697-714. https://doi. org/10.1177/1077801208317288

Grewal, D., \& Salovey, P. (2006). Inteligencia emocional. Mente y cerebro, 16(1), 10-20.

Hernández, A., Beauring, T., Berner, E., Blum,R., Florenzano, R., Lammers,C., Serrano, C., Valdés,M. (1996). Familia adolescencia: indicadores de salud, Manual de aplicación de instrumentos. Fundación W.K. Kellogg.

http://www.rehueong.com.ar/sites/default/files/Familia\%20 y\%20Adolescencia\%20Indicadores\%20de\%20Salud.pdf

Joseph, D. L.; Jin, J.; Newman, D. A. y O’Boyle, E. H. (2015). Why does self-reported emotional intelligence predict job performance?: A meta-analytic investigation of mixed EI. Journal of Applied Psychology, 100(2), 298-342. https://doi.org/10.1037/ a0037681
Martínez-González, R. A. (2009). Programa-Guía para el desarrollo de competencias emocionales, educativas y parentales. Secretaría General Técnica, Ministerio de Sanidad y Política Social. https://www.mscbs.gob.es/va/ssi/familiasInfancia/docs/programa2009_1.pdf

McCubbin H. I., Patterson J. M. et al. (1981). Family Inventory of Life Events and Changes (FILE), form A. St. Paul, University of Minnesota.

Méndez, P., y Barra, E. (2008). Apoyo social percibido en adolescentes infractores de ley y no infractores. Psykhe (Santiago), 17(1), 59-64. http://dx.doi.org/10.4067/S071822282008000100006

Mestre, V. (2014). Prosocialidad: evaluación e intervención. Propuestas de futuro. Revista Mexicana de Investigación en Psicologia, 195-201. http://mail.revistamexicanadeinvestigacionenpsicologia.com/index.php/RMIP/article/view/197

Ministerio de Educación (2020). Guía Juntos aprendemos y nos cuidamos (Régimen Sierra-Amazonía 2020-2021). Plan Educativo Sección 5: Socioemocional. https://educacion.gob.ec/wp-content/ uploads/downloads/2020/09/Seccion-5_Socioemocional.pdf

Moos, R. H., Moos, B. S. y Trickett, E. J. (1995). Manual de Escalas de clima social ( $4^{\mathrm{a}}$ ed.). TEA

Olson, D.H.; McCubbin, H.I. et al. (Eds.) (1982). Family inventories: Inventories used in a national survey of families across the family life cycle. Ed. St. Paul, University of Minnesota.

Pena, M. y Repetto, E. (2008). Estado de la investigación sobre Inteligencia Emocional en España en el ámbito educativo. Electronic Journal of Research in Educational Psychology, 6(15), 400-420. https://doi.org/10.25115/ejrep.v6i15.1284

Pérez Escoda, N., y Filella Guiu, G. (2019). Educación emocional para el desarrollo de competencias emocionales en niños y adolescentes. Praxis $\mathcal{E}$ Saber, 10(24), 23-44. https://doi. org/10.19053/22160159.v10.n25.2019.8941

Polo del Río, M. I., Fajardo, F., Martín, E., Gómez-Carroza, T. y León del Barco, B. (2012). Aceptación/rechazo parental en una muestra de adolescentes. Diferencias según sexo y edad. International Journal of Developmental and Educational Psychology, 1(1), 329-335. http://www.redalyc.org/articulo. oa?id=349832342033

Rodrigo, M. J., García, M., Máiquez, M. L., Rodríguez, B., y Padrón, I. (2008). Estrategias y metas en la resolución de conflictos cotidianos entre adolescentes, padres y madres. Infancia y Aprendizaje, 31(3), 347-362. https://doi.org/10.17060/ ijodaep.2014.n1.v6.720

Sanmartín, R., Gonzálevez, C. y Vicent, M. (2018). Inteligencia emocional en alumnado de formación profesional. Diferencias en función del curso, del género y de la edad. Educar, 54(1), 229-245. https://raco.cat/index.php/Educar/article/ view/331867

Salovey, P. E., \& Sluyter, D. J. (1997). Emotional development and emotional intelligence: Educational implications. Basic Books. https://psycnet.apa.org/record/1997-08644-000

Sánchez-Núñez, M., y Latorre Postigo, J. M. (2012). Inteligencia emocional y clima familiar. Behavioral Psychology/Psicología Conductual, 20(1).

Valiente, C., Árguedas-Morales, M. et al. (2020). Fortaleza psicológica adolescente: relación con la inteligencia emocional y los valores. Aula Abierta, 49(4), 385-394. https://doi. org/10.17811/rifie.49.4.2020.385-394

Zicavo, N., Palma, C., y Garrido, G. (2012). Adaptación y validación del Faces-20-ESP: Reconociendo el funcionamiento familiar en Chillán, Chile. Revista Latinoamericana de Ciencias Sociales, Niñez y Juventud, 10(1), 219-234. http://hdl.handle. net/20.500.11907/1026 
